\section{Pulmonary hypertension:}

\section{diagnosis and treatment}

Charlie Elliot MB MRCP, Specialist Registrar

David G Kiely MD FCCP, Consultant

Respiratory Physician

Sheffield Pulmonary Vascular Disease Unit, Royal Hallamshire Hospital, Sheffield

Clin Med 2004;4:211-15

Pulmonary hypertension $(\mathrm{PH})$ is a challenging condition to diagnose, accurately classify and treat. There is often a delay from the onset of symptoms to diagnosis of up to three years. ${ }^{1,2}$ The diagnostic process currently requires invasive investigations. Treatments are now effective, but often complex. Until the advent of transplantation in the 1980s there was no specific treatment for $\mathrm{PH}$. The last two

Table 1. World Health Organization reclassification of pulmonary hypertension (PH) (2003).

- Pulmonary arterial hypertension

- $\mathrm{PH}$ with left heart disease*

- $\mathrm{PH}$ with lung disease and/or hypoxaemia*

- $\mathrm{PH}$ due to thrombotic and/or embolic disease

- Miscellaneous group

${ }^{*}$ In these instances, treatment is best aimed at the underlying disease. These patients do not usually require specialist assessment, but those with certain forms of $\mathrm{PH}$ with lung disease may respond to targeted pulmonary vascular therapy. If there is doubt, cases can be discussed with the local PH centre. decades have seen the development of novel therapies which improve symptoms and survival of patients with $\mathrm{PH}$.

$\mathrm{PH}$ is defined as a mean pulmonary artery pressure of $25 \mathrm{mmHg}$ or above at rest or $30 \mathrm{mmHg}$ or above on exercise. ${ }^{1}$ The World Health Organization recently reclassified $\mathrm{PH}$ and identified five major groups (Table 1), demonstrating the importance of identifying the cause of $\mathrm{PH}$ in deciding on treatment. Patients with chronic thromboembolic pulmonary hypertension (CTEPH) can be cured by surgery. Those with pulmonary arterial hypertension (PAH) (Table 2) can be improved with selective pulmonary arterial vasodilators, but these drugs can precipitate pulmonary oedema in patients with pulmonary venous hypertension.

\section{Epidemiology}

PAH and CTEPH have an estimated prevalence of 30-50 cases per million. Idiopathic pulmonary hypertension (IPH), a form of PAH previously called primary pulmonary hypertension, is a severe, progressive disease with an incidence of 1-2 cases per million per year and three times more common in women. ${ }^{2}$ Without targeted pulmonary vascular therapy, it has a median survival of 2.8 years from diagnosis.

\section{Making the diagnosis}

The non-specific nature of the symptoms and the subtle nature of the signs of pul- monary vascular disease often delay diagnosis. Making a diagnosis therefore requires an awareness of the possibility of $\mathrm{PH}$ and recognition of the prevalence of pulmonary vascular disease in patients with associated conditions such as systemic sclerosis $(10-20 \%),{ }^{3}$ sickle cell disease (20\%), HIV infection (0.5-2\%), portal hypertension (1-2\%), pulmonary embolic disease and congenital heart disease.

\section{Clinical features}

The cardinal symptom of $\mathrm{PH}$ is breathlessness because the right heart is unable to generate a sufficient increase in cardiac output on exercise. Initially, this may be mild but it is progressive and later may be accompanied by chest pains (similar to angina) and syncope, often on exercise. Syncope usually reflects a low cardiac output and indicates severe disease. As right heart failure develops, abdominal distension, general fatigue and ankle swelling may appear - ankle swelling occurs only late in the natural history of the disease.

Signs on examination may initially be few but are more readily seen with disease progression (and after the diagnosis is made) (Table 3 ).

\section{Investigations}

Key basic investigations include an ECG and/or chest X-ray (CXR) which are abnormal in approximately $80 \%$ of patients. Pulmonary function tests may suggest an alternative diagnosis. The most common investigation suggesting $\mathrm{PH}$ is a trans-thoracic echocardiogram.

Table 3. Clinical signs in advanced pulmonary hypertension.

Table 2. Pulmonary arterial hypertension (PAH).

\begin{tabular}{ll} 
Idiopathic pulmonary hypertension & Sporadic \\
& Familial \\
\hline Related to: & Collagen vascular disease \\
& Portal hypertension \\
& Congenital heart disease \\
& HIV infection \\
& Drugs/toxins
\end{tabular}

Persistent pulmonary hypertension of the newborn

PAH with significant venous or capillary involvement
- Tachycardia

- Elevated JVP

- RV heave

- Pansystolic murmur

- Loud P2

- Hepatomegaly - pulsation

- Ascites

- Peripheral oedema

JVP = jugular venous pressure; $\mathrm{RV}=$ right ventricular. 
This allows estimation of the systolic pulmonary artery pressure from the tricuspid regurgitant jet using Doppler echocardiography. Dilated right-sided chambers or paradoxical septal motion may also be observed, reflecting high right-sided pressures. Echocardiography may also help to identify the cause (valvular heart disease, left ventricular dysfunction, intracardiac shunts).

If significant $\mathrm{PH}$ is suspected, the patient should be referred to a specialist centre for further investigation and assessment. Patients with PH due to leftsided heart problems and underlying

Table 4. Aims of specialist assessment.

- Confirm or exclude diagnosis of PH

- Assess disease severity

- Establish disease aetiology

- Identify patients with potentially operable CTEPH

- Institute management plan with patient education and agreement

CTEPH = chronic thromboembolic pulmonary hypertension; $\mathrm{PH}=$ pulmonary hypertension

Table 5. Investigations.

$\begin{array}{ll}\text { Imaging } & \text { Chest X-ray } \\ & \text { Ventilation perfusion } \\ & \text { scanning } \\ & \text { HRCT lungs } \\ & \text { Contrast helical CT } \\ & \text { pulmonary arteries } \\ & \text { Magnetic resonance } \\ & \text { angiography } \\ & \text { Pulmonary angiogram (in } \\ & \text { selected cases) } \\ \text { Pulmonary } & \text { Arterial blood gases } \\ & \text { Lung function } \\ & \text { Nocturnal oxygen saturation } \\ & \text { monitoring } \\ & \text { Exercise test (6-minute } \\ & \text { walk/shuttle) } \\ \text { Cardiology } & \text { ECG } \\ & \text { Echocardiography } \\ & \text { Cardiac catheterisation } \\ \text { Blood } & \text { Routine haematology and } \\ & \text { biochemistry } \\ & \text { Thrombophilia screen } \\ & \text { Autoimmune screen } \\ & \text { HIV testing }\end{array}$

$\mathrm{CT}$ = computed tomography;

$\mathrm{HRCT}=$ high-resolution $\mathrm{CT}$. respiratory disease are most effectively treated by addressing the underlying cause (Table 1).

\section{Further assessment}

Following referral, invasive investigation (right-heart catheterisation with a vasodilator challenge) is usually necessary (Table 4). Further investigations are shown in Table 5.

In Sheffield 'one-stop' and 'two-stop' assessment services are offered. Many patients prefer the 'two-stop' service which allows them to meet the team prior to admission for invasive investigation.

\section{Two-stop assessment}

A number of non-invasive investigations are carried out on the initial visit of the 'two-stop' assessment. Following this, patients are usually well informed and able to consider the potential implications of a diagnosis of PH. Supplementary imaging investigations and cardiac catheterisation are performed during their second visit. Investigation results are then reviewed by a multidisciplinary team, usually allowing a clear pulmonary vascular diagnosis.

\section{Screening}

There is a $10-20 \%$ prevalence of $\mathrm{PH}$ in systemic sclerosis. We have identified patients with early disease (Fig 1) using a pragmatic screening approach. Patients at risk of CTEPH can be identified using the criteria in Table 6. Family members of patients with IPH at increased risk of developing $\mathrm{PH}$ may be identified with the aid of genetic screening or exercise testing, although the low penetrance $(10-20 \%)$ of the familial form of $\mathrm{PH}$ (autosomal dominant inheritance) means that this must be approached sensitively. Genetic linkage studies have mapped a disease locus, designated PPH1, to the long arm of chromosome 2; subsequently, germline mutations in the bone morphogenetic protein receptor have been identified in a subset of families with IPH.

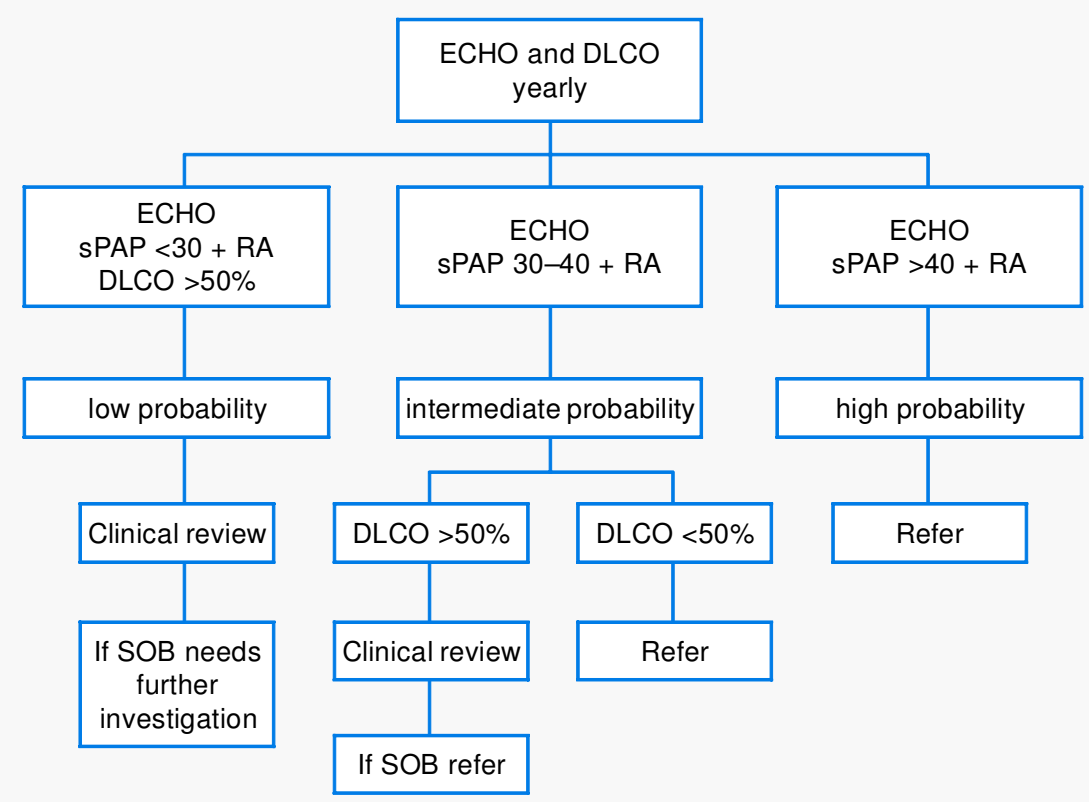

Please refer all patients with high probability of pulmonary hypertension, or patients with intermediate probability who are symptomatic or have DLCO $<50 \%$. Note: sPAP should not include estimate of RA pressure, expressed as SPAP + RA ( $R A=10 \mathrm{mmHg}$, usually an overestimate for purposes of screening).

Fig 1. Sheffield Pulmonary Vascular Disease Unit screening approach for pulmonary hypertension using yearly echocardiography $(\mathrm{ECHO})$ and transfer factor in systemic sclerosis (DCLO = transfer factor; sPAP = systemic pulmonary artery pressure; $\mathrm{RA}=$ right atrial pressure; $\mathrm{SOB}=$ shortness of breath). 


\section{Treatment}

Treatment should ideally be instituted in a specialist centre, a view shared by patient groups (eg the Pulmonary Hypertension Association (PHA-UK)). There are five nationally designated centres in the UK (Sheffield, London, Cambridge, Newcastle and Glasgow) and a centre in Ireland; they have experience with the initiation, continued use and monitoring of targeted drug treatments and the infrastructure necessary for patient education and support. They also have expertise in patient selection for, and the timing of, surgical interventions.

Treatment is demanding but often rewarding. The right ventricle has a huge potential to remodel if pulmonary vascular resistance can be reduced by surgery or medical therapy. This is illustrated in Figs 2 and 3 which demonstrate improvement in both ECG and CXR following one year's treatment with intravenous iloprost.

Standard supportive treatments for $\mathrm{PH}$ include warfarin, diuretics, digoxin and oxygen therapy.

\section{Targeted pulmonary vascular treatment}

The number of targeted pulmonary vascular therapies for PAH has increased dramatically over the past five years following a number of phase 3 randomised controlled trials (RCT). These treatments are directed at the pulmonary arterial vascular bed and have also been

Table 6. Patients at increased risk of chronic thromboembolic pulmonary hypertension.

\section{Massive and submassive PE}

Diagnosis of $\mathrm{PE}$ and:

- sPAP >50 mmHg on admission echocardiogram

- $\mathrm{sPAP}>40 \mathrm{mmHg}$ on echocardiogram at six weeks

Ongoing dyspnoea following PE or New onset dyspnoea with a previous history of DVT or PE

DVT = deep venous thrombosis;

sPAP = systolic pulmonary artery pressure;

$\mathrm{PE}=$ pulmonary embolism. extensively used off-label in other categories of the disease, including CTEPH. The drugs include epoprostenol (prostacyclin) and its analogues, iloprost and treprostinil, and the endothelin antagonist bosentan. These therapies are expensive, costing $\mathfrak{2} 20,000-35,000$ per patient-year. More recently, in a few small studies the phosphodiesterase inhibitor sildenafil has reduced pulmonary artery pressure; it is currently the focus of a phase 3 multicentre study.

\section{Complex treatments}

Intravenous epoprostenol improves life expectancy, haemodynamics, six-minute walk time (frequently used as a surrogate marker of mortality) and quality of life in patients with severe IPH (New York Heart Association (NYHA) class III and IV). ${ }^{4}$ However, continuous infusion via a Hickman line is required, posing a risk of life-threatening infection.

Iloprost is a more stable analogue of epoprostenol. It can be delivered via a
Hickman line or a nebuliser. Using the latter, it was an effective treatment for $\mathrm{PAH}$ in the context of a multicentre RCT. ${ }^{5}$ However, its short half-life means that patients need to nebulise 6-9 times per day.

Treprostinil, another prostaglandin analogue, administered subcutaneously, has also been shown to be efficacious in the largest RCT ever conducted in $\mathrm{PH} .{ }^{6}$ Problems include infusion site pain.

\section{Oral treatments}

Oral treatment options include calciumchannel antagonists. Despite a lack of RCT data they are advocated as an effective therapy, although in practice their efficacy is limited to a small minority of patients with IPH with a positive 'vasodilator response' (a fall of greater than $20 \%$ in mean pulmonary artery pressure following administration of a pulmonary vasodilator such as nitric oxide or epoprostenol). Beraprost, an orally active prostaglandin, is effective

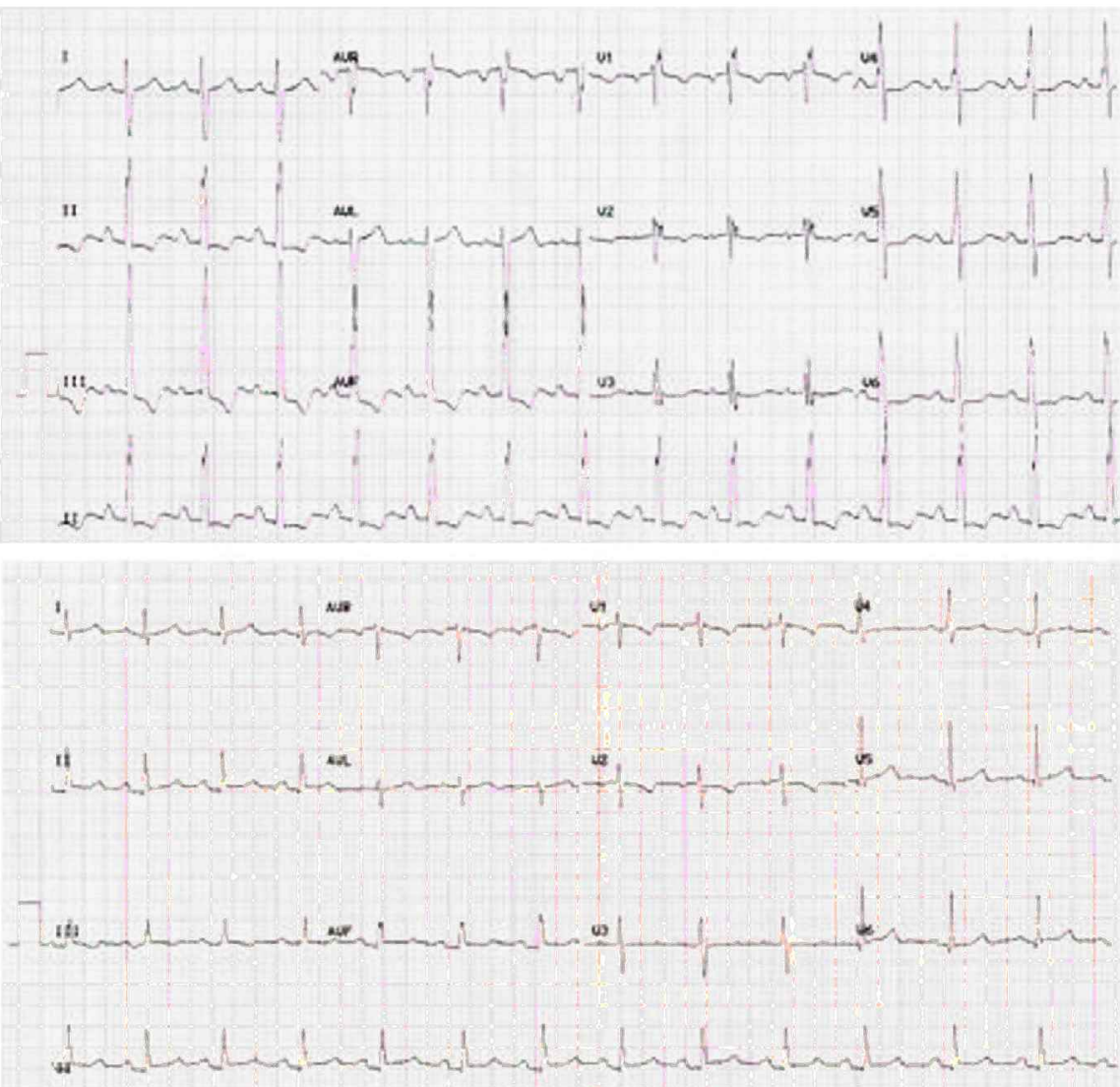

Fig 2. ECG improvement following intravenous iloprost therapy for idiopathic pulmonary hypertension. 

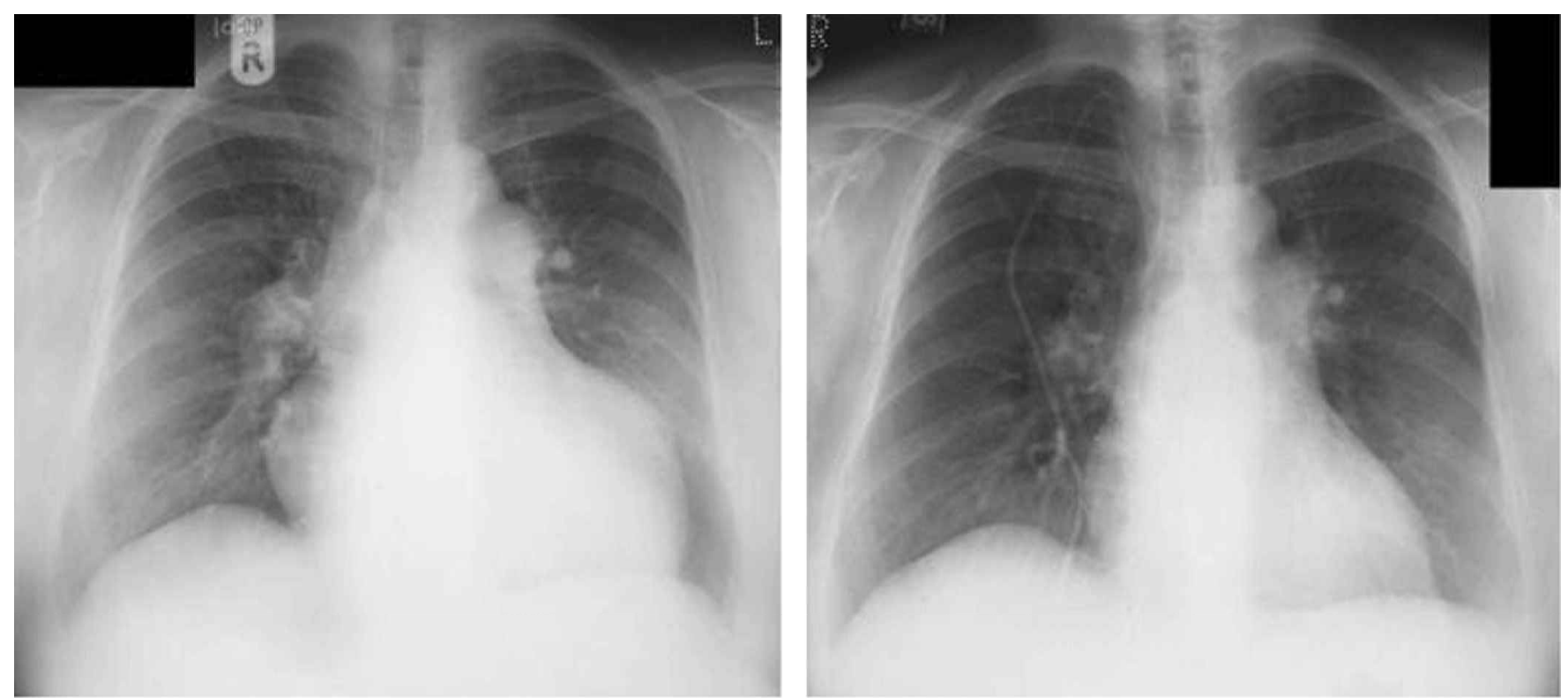

Fig 3. Radiographic improvement following intravenous iloprost therapy for idiopathic pulmonary hypertension.

although it has been investigated only in patients with milder disease (NYHA class II and III). ${ }^{7}$ The endothelin receptor antagonist bosentan currently represents the only licensed oral treatment for PAH in the UK. It improves the symptoms of $\mathrm{PH}$ and physiological markers of disease severity. ${ }^{8}$ The results of a phase 3 study examining the potential role of sildenafil are eagerly awaited.

It is anticipated that in the future patients will receive combination therapy - a number of reports suggest that combination therapy may be efficacious.

\section{Surgical options}

Patients identified as having thromboembolic disease should be assessed for pulmonary endarterectomy, which offers a return to an almost normal quality and quantity of life in a proportion of these patients. The operative mortality in experienced hands is $5-10 \%$, comparing favourably with a five-year survival of less than $10 \%$ once a mean pulmonary artery pressure of above $50 \mathrm{mmHg}$ is exceeded in this form of $\mathrm{PH}$.

Heart-lung or lung transplantation is usually reserved for patients deteriorating on pulmonary vascular directed therapy.

Another option is atrial septostomy which involves making a small incision in the interatrial septum. This can be performed at cardiac catheterisation and appears to be an effective way of offloading the right ventricle, although its role is as yet not clearly defined.

\section{Conclusions}

The diagnosis of $\mathrm{PH}$ is often made at a mid to late stage in the course of the disease. In a study carried out by PHA-UK fewer than $5 \%$ of patients present as an acute emergency. Only 30\% of patients go to see their general practitioner within one month of experiencing symptoms, most waiting over six months. Over $40 \%$ of patients go on to see four or more doctors before a diagnosis of $\mathrm{PH}$ is made. With the advent of effective therapies and an increased awareness of $\mathrm{PH}$, it is hoped that early diagnosis and timely referral to one of the UK specialist centres will improve the prognosis and quality of life for these patients.

\section{Conflicts of interest}

Dr Kiely has participated in clinical trials examining the efficacy of treprostinil, nebulised iloprost and sildenafil as treatments for PH. He currently sits on an advisory

\section{Key Points}

Consider pulmonary hypertension as a possible diagnosis in patients with
unexplained breathlessness and in high risk groups such as patients with
systemic sclerosis and past history of pulmonary embolism.

The symptoms of pulmonary hypertension are non-specific and clinical signs often subtle.

Echocardiography is a useful tool in making a diagnosis of pulmonary hypertension

Effective drug and surgical treatments now exist for certain forms of pulmonary hypertension.

Nationally designated centres are available to give advice and manage patients with pulmonary hypertension.

KEY WORDS: Pulmonary circulation, pulmonary vascular disease, idiopathic pulmonary hypertension, chronic thromboembolic pulmonary hypertension 
board for Actelion Pharmaceuticals, the manufacturers of bosentan, and on the TRAX PMS Advisory Board. He has received payment from pharmaceutical companies for organising educational seminars.

\section{References}

1 British Cardiac Society Guidelines and Medical Practice Committee, approved by the British Thoracic Society and British Society of Rheumatology. Recommendations on the management of pulmonary hypertension in clinical practice. Heart 2001;86(Suppl 1):i1-i13.

2 Rich S, Dantzker DR, Ayres SM, Bergofsky $\mathrm{EH}$ et al. Primary pulmonary hypertension. A national prospective study. Ann Intern Med 1987;107:216-23.

3 Stupi AM, Steen VD, Owens GR, Barnes EL et al. Pulmonary hypertension in the CREST syndrome variant of systemic sclerosis. Arthritis Rheum 1986;29:515-24.

4 Barst RJ, Rubin LJ, Long WA, McGoon MD et al. A comparison of continuous intravenous epoprostenol (prostacyclin) with conventional therapy for primary pulmonary hypertension. The Primary Pulmonary Hypertension Study Group. N Engl J Med 1996;334:296-302.

5 Olschewski H, Simonneau G, Galie N, Higenbottam $\mathrm{T}$ et al. Inhaled iloprost for severe pulmonary hypertension. $N$ Engl $J$ Med 2002;347:322-9.

6 Simonneau G, Barst RJ, Galie N, Naeije R et al. Continuous subcutaneous infusion of treprostinil, a prostacyclin analogue, in patients with pulmonary arterial hypertension: a double-blind, randomized, placebocontrolled trial. Treprostinil Study Group. Am J Respir Crit Care Med 2002;165:800-4.

7 Galie N, Humbert M, Vachiery JL, Vizza $\mathrm{CD}$ et al. Effects of beraprost sodium, an oral prostacyclin analogue, in patients with pulmonary arterial hypertension: a randomized, double-blind, placebo-controlled trial. J Am Coll Cardiol 2002;39:1496-502.

8 Rubin LJ, Badesch DB, Barst RJ, Galie N et al. Bosentan therapy for pulmonary arterial hypertension. N Engl J Med 2002;346: 896-903.

\section{Suspected}

\section{pulmonary embolism}

Andrew C Miller DM DPhil FRCP, Consultant Physician, Chest Clinic, Mayday University Hospital, Croydon

Clin Med 2004;4:215-19

In June 2003 the British Thoracic Society (BTS) published 'Guidelines for the management of suspected acute pulmonary embolism.' Its title acknowledges the clinical experience that pulmonary embolism (PE) is not easy to diagnose. A frequent thought sequence, particularly of junior physicians, is:

1 Unrecognised PE can be fatal.

2 Common symptoms in PE are chest pain, and/or dyspnoea and/or haemoptysis.

3 Therefore any patient with the above symptom(s) may have PE.

4 If there is any doubt, it is negligent not to investigate for PE.

This fear of missing PE may account for physicians being better at excluding PE (wrong in only $10 \%$ of cases) than in diagnosing it (wrong in $30 \%$ of cases) on clinical grounds. ${ }^{2}$ In all major studies of suspected PE, it is excluded in $65-85 \%$ of cases. Unfortunately, these studies rarely discuss the nature of the true non-PE diagnosis and whether this could have been ascertained earlier; this could be a fruitful and revealing local audit project. ${ }^{3}$

\section{Symptoms in pulmonary embolism and other acute chest illnesses}

The commonest alternative diagnoses are shown in Table 1. The table demonstrates how assessing presenting $\operatorname{symptom}(\mathrm{s})$ in the light of chest anatomy can focus thinking towards the probable diagnosis. PE is a disease of the pulmonary circulation, hence indirectly affecting the lung parenchyma, whereas symptoms such as cough and haemoptysis may instead arise from the airways. Junior doctors often assume that noncardiac chest pain might be due to PE, unaware that the lung has no pain fibres. $P E$ can cause chest pain only if there are peripheral parenchymal changes spreading outside the lung to the (very sensitive) parietal pleura. Where chest radiograph (CXR) shows normal lung parenchyma, acute pleuritic chest pain most likely arises from the parietal pleura (perhaps with an effusion) or chest wall, making PE unlikely.

Dyspnoea, tachypnoea and hypoxia are

\section{Key Points}

Symptoms compatible with pulmonary embolism (PE) are not by themselves sufficient justification for organising special imaging tests

The correct diagnosis can often be reached by careful consideration of (a) clinical information (b) knowledge of chest anatomy, and (c) a good quality departmental chest X-ray

A combination of low clinical probability plus low/negative D-dimer makes further imaging and anticoagulation unnecessary

Computed tomographic pulmonary angiography should now be considered the principal imaging test for $\mathrm{PE}$

Suspected massive PE justifies (a) immediate advice from a consultant physician (b) emergency echocardiogram, and (c) consideration of early thrombolysis

Decisions about duration of anticoagulation should be made by three (not 6) months, both in PE and deep vein thrombosis

KEY WORDS: anticoagulation, clinical probability, computed tomographic pulmonary angiography, D-dimer, massive, thrombolysis. 